\title{
Acacia nilotica (Babool) leaf extract mediated size-controlled rapid synthesis of gold nanoparticles and study of its catalytic activity
}

\author{
Rakhi Majumdar, Braja Gopal Bag* and Nabasmita Maity
}

\begin{abstract}
The leaf extract of Acacia nilotica (Babool) is rich in different types of plant secondary metabolites such as flavanoids, tannins, triterpenoids, saponines, etc. We have demonstrated the use of the leaf extract for the synthesis of gold nanoparticles in water at room temperature under very mild conditions. The synthesis of the gold nanoparticles was complete in several minutes, and no extra stabilizing or capping agents were necessary. The size of the nanoparticles could be controlled by varying the concentration of the leaf extract. The gold nanoparticles were characterized by HRTEM, surface plasmon resonance spectroscopy, and X-ray diffraction studies. The synthesized gold nanoparticles have been used as an efficient catalyst for the reduction of 4-nitrophenol to 4-aminophenol in water at room temperature.
\end{abstract}

Keywords: Gold nanoparticle; Green synthesis; Acacia nilotica; Polyphenols; Catalytic reduction

\section{Background}

The synthesis of gold nanoparticles (AuNPs) and their utilization in diversified areas such as catalysis, drug delivery, biodiagnostics, medicine, and electronics have become an area of tremendous investigations during the last two decades because of their unique optical, physical, chemical, and magnetic properties compared to the bulk solids [1-4]. Among various synthetic methods, the solution phase synthesis involving the reduction of $\mathrm{Au}$ (III) to $\mathrm{Au}(0)$ by plant extracts has gained profound significance in recent years because of the renewable and nontoxic nature of the plant extracts, eco-friendly aqueous medium, and mild reaction condition [5]. Moreover, this method becomes more advantageous over other synthetic methods since the plant extract itself acts as a stabilizer, and no additional stabilizers or capping agents are needed [6]. The extracts of Punica granatum [7], Breynia rhamnoides [8], Saraca indica [9], Piper betle [10], etc. have been utilized for the synthesis of AuNPs. During our investigations on the utilization of triterpenoids (C30s) as renewable functional nanoentities [11-14], it occurred to us that the medicinally important

\footnotetext{
*Correspondence: braja@mail.vidyasagar.ac.in

Department of Chemistry and Chemical Technology, Vidyasagar University,
} Midnapore 721102, WB, India

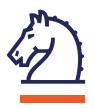

\section{Methods}

Materials

$\mathrm{Au}(\mathrm{III})$ solution, $\mathrm{HAuCl}_{4}$, was purchased from Sisco Research Laboratory, Mumbai, India, and used without further purification. $\mathrm{HAuCl}_{4}(35.4 \mathrm{mg})$ was dissolved in distilled water $(10 \mathrm{~mL})$ to obtain a $10.42-\mathrm{mM} \mathrm{Au}(\mathrm{III})$ stock solution. The leaves of $A$. nilotica were collected from the Vidyasagar University, Midnapore, India campus. Doubled distilled water was used for experimental purposes. 


\section{Preparation of the $A$. nilotica leaf extract}

Dried and finely powdered A. nilotica leaf (2.9 g) was suspended in ethanol $(30 \mathrm{~mL})$ and stirred magnetically at room temperature for $1 \mathrm{~h}$ and then filtered. Volatiles of the greenish filtrate was removed under reduced pressure to afford a sticky solid (0.390 g) that was purified by column chromatography ( $\mathrm{Si}$ gel, 100 to 200 mesh) using 30\% methanol-ethyl acetate as the eluant affording a greenish solid $(0.3 \mathrm{~g})$. The column-purified leaf extract $(0.005 \mathrm{~g})$ was suspended in distilled water $(10 \mathrm{~mL})$ and sonicated in an ultrasonicator bath for $10 \mathrm{~min}$ to get a semitransparent solution $\left(500 \mathrm{mgL}^{-1}\right)$.

\section{Synthesis of nanoparticles}

Aliquots of $\mathrm{Au}(\mathrm{III})$ solution (0.16 mL, $10.42 \mathrm{mM}$ each) were added dropwise to the leaf extract solution (containing polyhydroxy aromatic compounds) to prepare a series of stabilized AuNPs where the concentration of the leaf extract varied from 20 to $200 \mathrm{mg} \mathrm{L}^{-1}$, and the concentration of $\mathrm{Au}(\mathrm{III})$ was fixed at $0.42 \mathrm{mM}$. UVvisible measurements of the solutions were carried out after $3 \mathrm{~h}$ of $\mathrm{HAuCl}_{4}$, and A. nilotica leaf extract were mixed.

\section{Characterization}

Transmission electron microscopy of AuNPs was carried out in Tecnai $G^{2} 20$ instrument (FEI, Hillsboro, OR, USA). XRD patterns of the stabilized AuNPs were studied in Panalytical X'pert Pro diffractometer (Almelo, The Netherlands) with $\mathrm{Cu}-\mathrm{K} \alpha$ radiation $(\lambda=1.54 \AA$ Á). Mass spectral analysis of the left extract was carried out in Shimadzu GCMS QP 2100 plus (Kyoto, Japan). Shimadzu 1601 spectrophotometer was used for UV-visible spectroscopic measurements. The FTIR spectra of samples were recorded in Perkin Elmer FTIR Spectrum-II model (Waltham, MA, USA) using KBr pellet.

\section{Results and discussion}

A. nilotica (Babool) is a well-known medicinally important plant commonly found in the dry areas of Asia, Africa, and Australia. Various parts of the plant such as leaves, bark, roots, and fruits are used as medicine for the treatment of bronchitis, asthma, meningitis, hypoglycemia, pneumonia, sore throat, etc. Different types of plant secondary metabolites such as flavanoids, tannins, triterpenoids, saponines, etc. are present in the leaf extract of A. nilotica [15,16]. Au(III) having a high reduction potential can be reduced to $\mathrm{Au}(0)$ by the polyphenolic compounds present in the leaf extract with concomitant oxidation of the polyphenolic compounds to the corresponding quinones. The $\mathrm{Au}(0)$ atoms can collide with each other forming AuNPs, and the AuNPs can be stabilized by the resulting quinone derivatives and other coordinating ligands present in the plant extract. The stabilizing ligands present on the surface of the AuNPs will not allow further aggregation of the AuNPs. To test this, we treated the increasing concentrations of the aqueous leaf extract contained in vials with $\mathrm{HAuCl}_{4}$ solution (Figure 1). An appearance of pinkish red color within $5 \mathrm{~min}$ indicated the formation of AuNPs. On standing the solutions at room temperature for several hours, intensification of the color was observed and then the intensities remained constant for several months indicating the stabilities of the synthesized AuNPs.

\section{UV-visible spectroscopy studies}

Due to charge transfer interactions between metal and the chloro ligands of $\mathrm{HAuCl}_{4}$, the UV-visible spectrum showed two peaks at 220 and $288 \mathrm{~nm}$ (Figure 1a). On reaction with increasing concentration of the A. nilotica leaf extract, disappearance of these two peaks were observed with concomitant appearance of a SPR band between 533 to $529 \mathrm{~nm}$ region (Figure $1 \mathrm{~b}, \mathrm{c}, \mathrm{d}, \mathrm{e}, \mathrm{f}, \mathrm{g}$ ). The variation of color of the AuNP colloids has been reported arising due to the change in size, shape, composition, crystallinity, etc. [1]. In our present studies, a shift of the SPR band was observed from 533 to $529 \mathrm{~nm}$ and the intensities increased

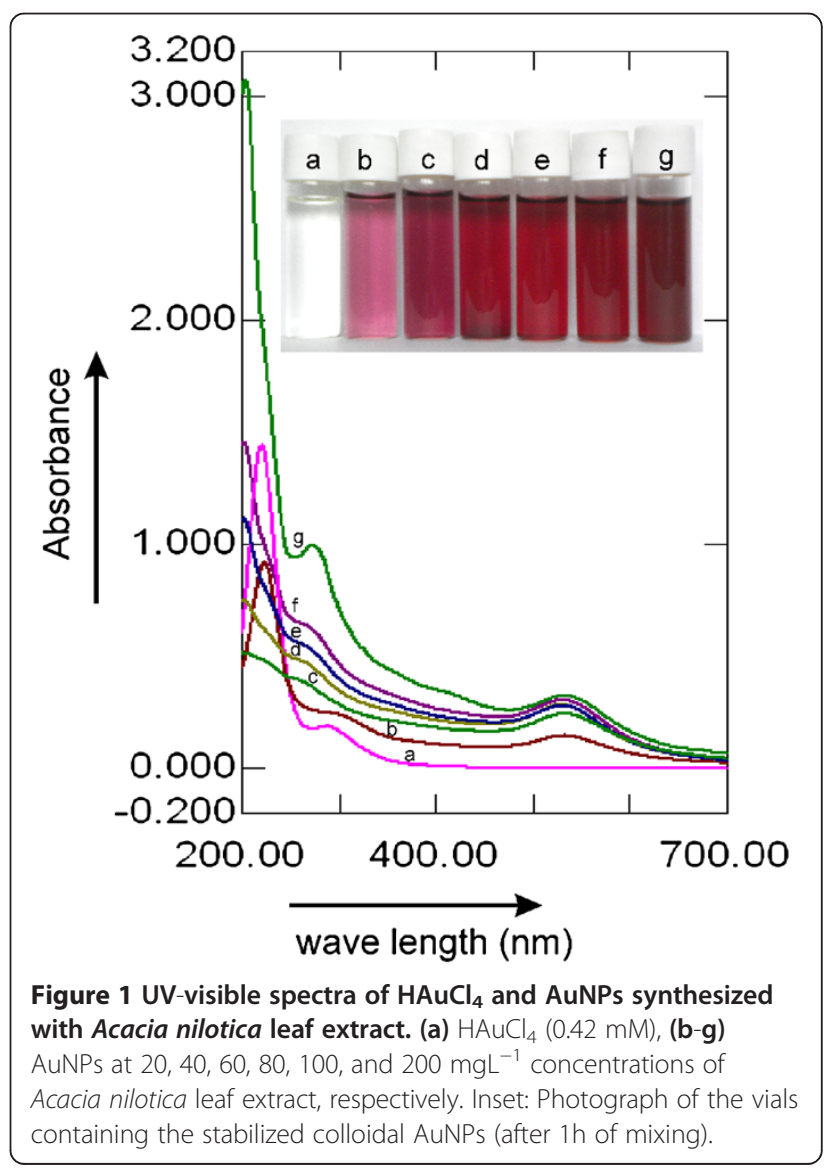


with the increasing concentration of the leaf extract from 20 to $200 \mathrm{mgL}^{-1}$. The strong peak at $272 \mathrm{~nm}$ at $200 \mathrm{mgL}^{-1}$ concentration is due to the formation of quinone moieties formed due to the oxidation of the phenolic compounds by $\mathrm{Au}(\mathrm{III})$ (Figure 1g).

\section{HRTEM, EDX, FTIR, and XRD studies}

The size distribution, shape, and morphology of the AuNPs formed at different concentrations of A. nilotica leaf extract were studied by HRTEM (see Figure 2 and Additional file 1: Figure S2). AuNPs of mostly spherical shapes of 6 to $12 \mathrm{~nm}$ size were observed. The average size of the AuNPs formed at $60 \mathrm{mgL}^{-1}$ concentration of the leaf extract was $12.24 \mathrm{~nm}$ (calculated from 77 particles, Figure 2a,b,c,d). At a higher concentration of the leaf extract $\left(200 \mathrm{mgL}^{-1}\right)$, the average particle size was $5.99 \mathrm{~nm}$ (calculated from 217 particles, Figure 2i,j,k,l). Elongated AuNPs (Figure 2e,g) were observed along with spherical particles (Figure 2f) at $100 \mathrm{mgL}^{-1}$ concentration of the leaf extract. The presence of the AuNPs held inside the organic matrix derived from the leaf extract was evident from Figure 2g.

With the increasing concentration of the leaf extract, smaller sized AuNPs can be effectively stabilized by the polyphenolic compounds and the oxidized quinones. As the steric bulk of the surrounding ligands of the AuNPs prevents aggregation, the size of the AuNPs were smaller at higher concentration of the leaf extract. Lattice fringes of the AuNPs with a d-spacing of $0.24 \mathrm{~nm}$ were observed from the HRTEM image (Figure 2c). The fringe spacing matched with the expected d-spacing of the [111] plane of face-centered cubic crystalline Au (JCPDS, no. 04-0784). Selected area electron diffraction (SAED) pattern obtained from a AuNP (Figure $2 \mathrm{~h}$ ) showed the diffraction rings from inner to outer associated with the [111], [200], [220], and [311] atomic planes of Au indicating the formation of crystalline gold nanoparticles. The elemental composition of the synthesized AuNPs was determined by energy dispersive X-ray analysis (EDX). Area profile analysis of the synthesized nanoparticles showed strong peaks of $\mathrm{Au}$

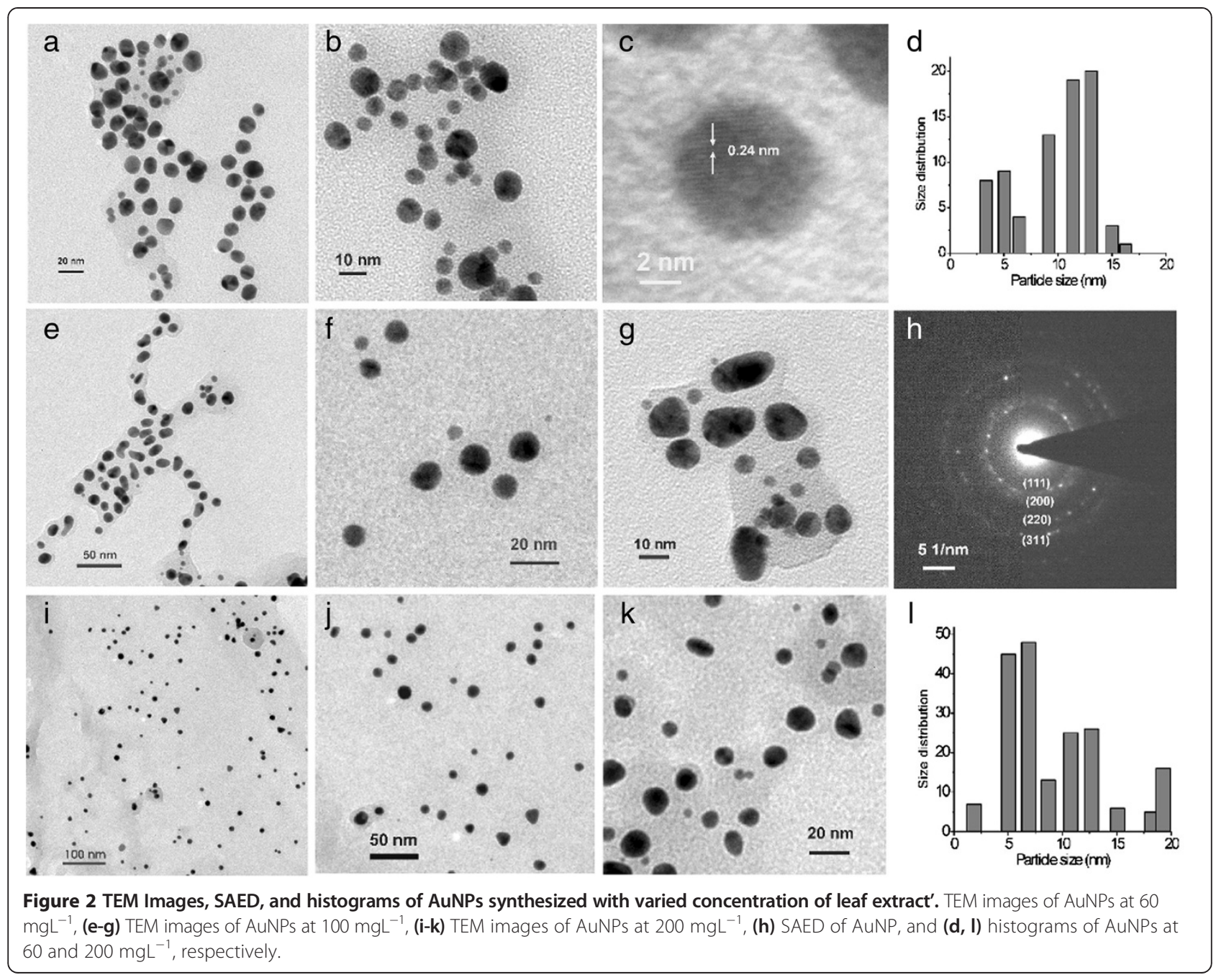




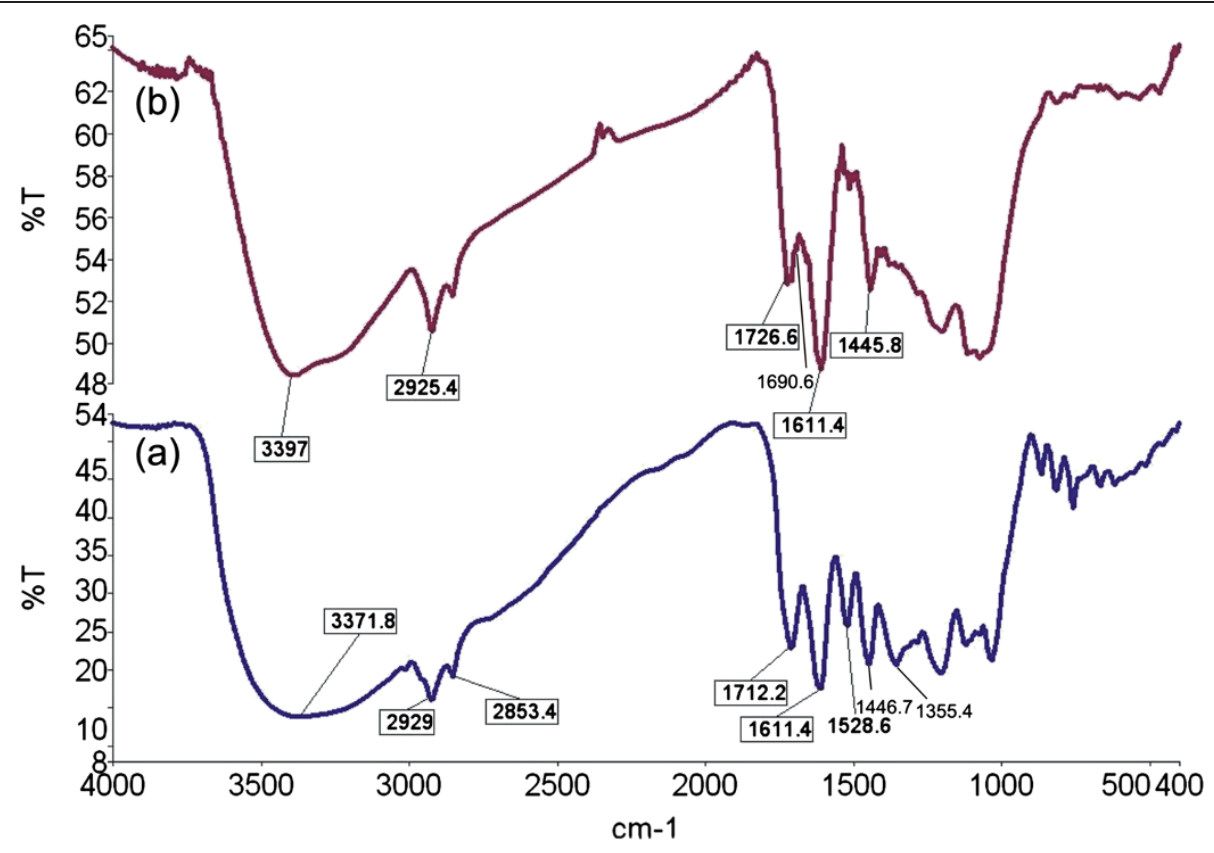

Figure 3 FTIR spectra of (curve a) Acacia nilotica leaf extract and (curve b) stabilized AuNPs.

(Additional file 1: Figure S3) confirming the formation of AuNPs. The presence of organic matrix was evident from characteristic carbon peak. The AuNP samples were coated over a glass plate, and X-ray diffraction analysis of the AuNPs was carried out after removal of the volatiles. The characteristic reflections of the planes (111), (200), (220), and (311) at $2 \theta=38.3^{\circ}, 44.3^{\circ}, 64.8^{\circ}$, and $77.8^{\circ}$, respectively, (Additional file 1: Figure S4) indicated the crystallinity of the metallic face-centered cubic AuNPs. These values are in agreement with the reported standards JCPDS file no. 04-0784 for crystalline gold. The comparatively greater peak intensity of the (111) plane is indicative of the predominant orientation of the (111) plane.
The FTIR spectra of the leaf extract of $A$. nilotica and the stabilized AuNPs were compared (Figure 3). The broad peak around $3,371 \mathrm{~cm}^{-1}$ in the FTIR spectrum of the leaf extract is due to the stretching vibration of the aliphatic and aromatic hydroxyl groups. Intermolecular hydrogen bonding among the hydroxyl groups was evident from the broadness of the peak. The peaks in the 1,611 to $1,400 \mathrm{~cm}^{-1}$ range $\left(1,611,1,528,1,447 \mathrm{~cm}^{-1}\right)$ are due to the presence of aromatic rings in the extract. The peak at $1,355 \mathrm{~cm}^{-1}$ is due to the in-plane bending of the $\mathrm{OH}$ groups. In the FTIR spectrum of the stabilized AuNPs, the stretching vibration due to the aliphatic and aromatic $\mathrm{OH}$ groups became narrower and shifted to $3,397 \mathrm{~cm}^{-1}$ due to weakening of

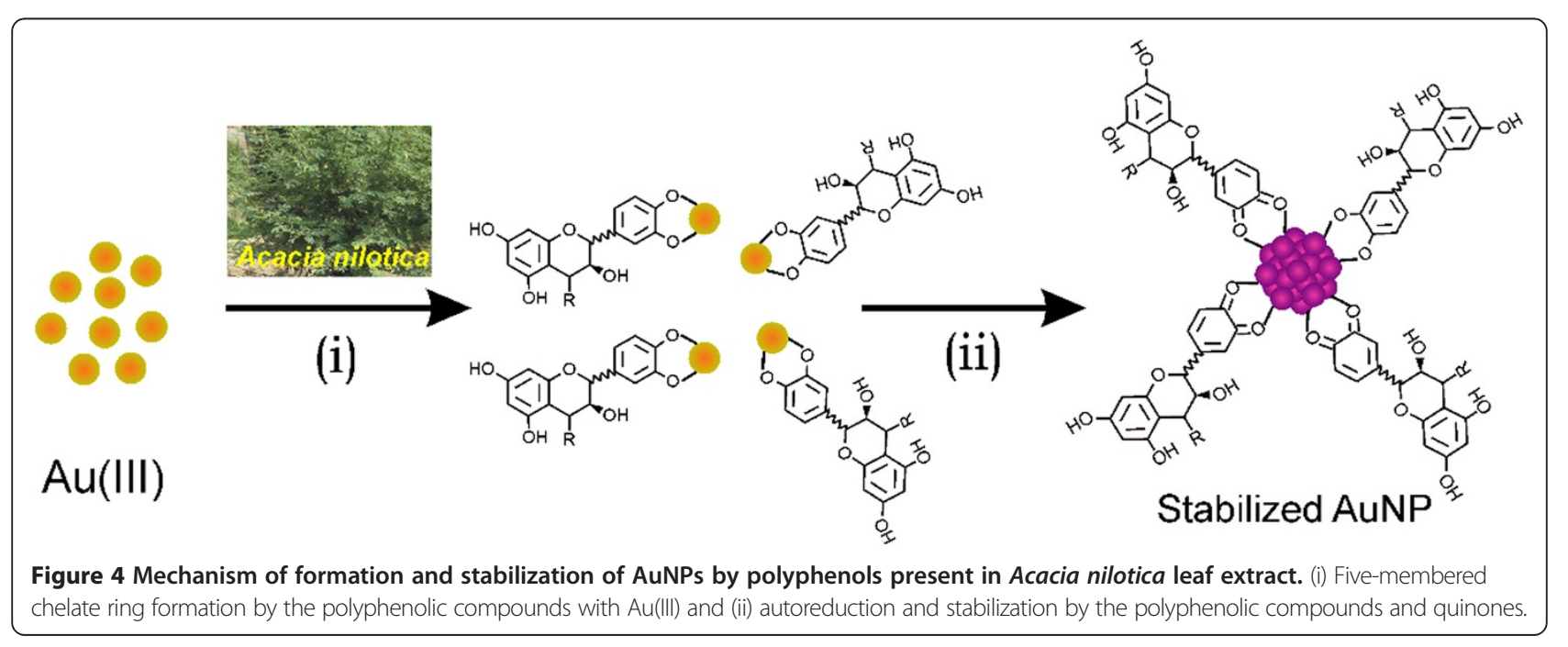


Table 1 Catalytic activity of colloidal AuNPs (synthesized freshly with $60 \mathrm{mgL}^{-1}$ of leaf extract)

\begin{tabular}{|c|c|c|c|c|c|}
\hline Sample number & $\begin{array}{c}\text { Concentration of } \\
\text { 4-nitrophenol }(\mathrm{mM})\end{array}$ & $\begin{array}{c}\text { Concentration of sodium } \\
\text { borohydride (mM) }\end{array}$ & $\begin{array}{c}\text { Volume of } \\
\text { colloidal AuNPs }\end{array}$ & $\begin{array}{c}\text { Reaction completion } \\
\text { time }(\mathrm{min})\end{array}$ & $\begin{array}{c}\text { Catalytic rate } \\
\text { constant }(k)\left(\min ^{-1}\right) \\
\end{array}$ \\
\hline 1 & 0.05 & 15.0 & 0.2 & 10 & 0.35 \\
\hline 2 & 0.05 & 15.0 & 0.4 & 6 & 0.42 \\
\hline 3 & 0.05 & 15.0 & 0.0 & No reduction & Not applicable \\
\hline
\end{tabular}

the intermolecular H-bonding. The in-plane bending frequency of $\mathrm{OH}$ group became much weaker in the case of the stabilized AuNPs (Figure 3). The appearance of a new peak at $1,691 \mathrm{~cm}^{-1}$ in the case of stabilized AuNPs is due to the formation of quinones.

\section{Mechanism of the formation of stabilized AuNPs}

The leaf extract of $A$. nilotica is rich in different types of plant secondary metabolites such as flavanoids, tannins, triterpenoids, saponines, etc. [15,16]. Evidence for the presence of polyphenolic compounds was obtained from the ferric chloride test (Additional file 1). Mass spectral analysis of the leaf extract carried out by us also supported the presence of the several polyphenolic compounds (Additional file 1: Figure S1) including quercetin $\left(\mathrm{M}^{+} 302\right)$, leucocyanidin $\left(\mathrm{M}^{+} 306\right)$, or their analogs. A schematic representation of the possible mechanism of the formation of AuNPs and their stabilization, based on leucocyanidin analogs as a representative framework, is shown in Figure 4. Ortho-dihydroxy compounds can form a five-membered chelate complex with $\mathrm{Au}(\mathrm{III})$. The chelated $\mathrm{Au}(\mathrm{III})$ can be reduced to $\mathrm{Au}(0)$ with concomitant oxidation of the ortho-dihydroxy compounds to quinones. Collision of the neighboring $\mathrm{Au}(0)$ atoms with each other lead to the formation of the AuNPs. The AuNPs can be stabilized by the polyphenolic compounds, quinones, as well as the other coordinating phytochemicals present in the leaf extract [17].

\section{Study of the catalytic activity of stabilized AuNPs}

To study the catalytic activity of the synthesized colloidal gold nanoparticles, the reduction of 4-nitrophenol (0.05 $\mathrm{mM})$ to 4-aminophenol by sodium borohydride (15 $\mathrm{mM})$ at room temperature was taken as a model system (Table 1) and the reaction was monitored by UV-visible spectroscopy (Figure 5) [17]. The absorption band of 4-nitorphenol at $318.5 \mathrm{~nm}$ shifted to $400 \mathrm{~nm}$ in the presence of sodium borohydride due to the formation of 4-nitrophenolate ion. No transformation was observed on standing the mixture at room temperature for several days. This is due to a very large kinetic barrier for the reduction reaction. Interestingly, on the addition of $A$. nilotica leaf extract-stabilized AuNPs (0.2 mL, synthesized with $60 \mathrm{mgL}^{-1}$ leaf extract) to the mixture, lowering of the intensity of the absorption peak at $400 \mathrm{~nm}$ was observed with concomitant formation of a new peak around $300 \mathrm{~nm}$ indicating the formation of 4-aminophenol. Complete disappearance of the 400-nm peak was observed within $10 \mathrm{~min}$ indicating completion of the reduction demonstrating the excellent catalytic activity of the synthesized colloidal AuNPs from the A. nilotica leaf extract. The change in intensity of the absorption maxima of 4-nitrophenol in alkaline medium (at $400 \mathrm{~nm}$ ) with time provides a reasonable tool for the study of its reduction kinetics. As the concentration of $\mathrm{BH}_{4}^{-}$was much higher (approximately 300-fold) than that of p-nitrophenol, its concentration remained practically constant during the reduction reaction and the reaction can be assumed as a

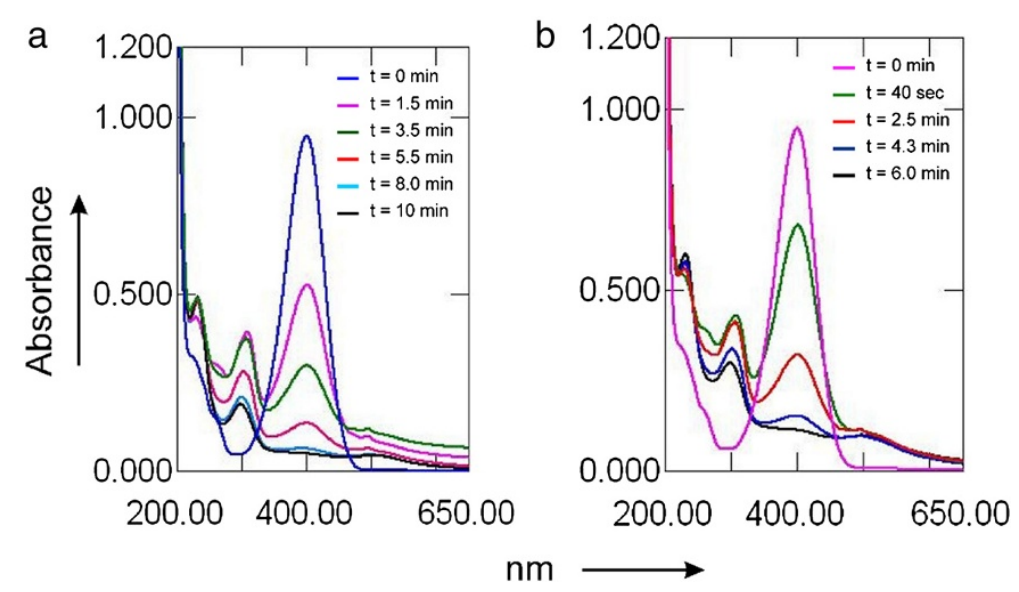

Figure 5 UV-visible spectra at different time intervals during the catalytic reduction of 4-nitrophenol to 4-aminophenol. Using (a) 0.2 and (b) $0.4 \mathrm{~mL}$ of stabilized AuNPs (synthesized with $60 \mathrm{mgL}^{-1}$ leaf extract). 
pseudo first order reaction. A good linear correlation between $\ln A$ vs time was obtained (where $A=$ absorbance of 4-nitrophenolate ion, Additional file 1: Figure S5), and the value of the catalytic rate constant $(k)$ was calculated to be $0.35 \mathrm{~min}^{-1}$. The observed rate constant was similar to that observed by us previously and others on similar systems $[7,8]$. Interestingly, on addition of double amount of stabilized AuNPs $\left(0.4 \mathrm{~mL}\right.$, synthesized with $60 \mathrm{mgL}^{-1}$ leaf extract) to the mixture, the rate of the reduction reaction became faster and the 400-nm peak completely disappeared within 6 min with concomitant formation of 4-aminophenol. Here also, a good linear correlation between $\ln A$ vs time was obtained and the value of the catalytic rate constant for this pseudo first order reaction was $0.42 \mathrm{~min}^{-1}$. It is believed that both 4-nitrophenol and the reducing agent $\mathrm{BH}_{4}^{-}$are adsorbed on the surface of the AuNPs, and the surface hydride ions are then transferred to 4-nitrophenol thereby facilitating the reduction reaction [18]. By comparing the rate constants of the two sets of reactions, it was obvious that with the larger volume of the catalyst, the rate of the reduction reaction increased due to the presence of larger number of reaction sites.

\section{Conclusions}

A very mild and efficient method for the green synthesis of colloidal gold nanoparticles has been demonstrated using the medicinally important $A$. nilotica leaf extract. According to our knowledge, this is the first report of the synthesis of AuNPs using A. nilotica leaf extract. The polyphenolic compounds present in the leaf extract act as an effective reducing agent as well as stabilizing agent, and AuNPs of 6 to $12 \mathrm{~nm}$ size were formed. Increasing the concentration of the leaf extract resulted in the formation of smaller sized nanoparticles as evident from HRTEM analysis. The synthesized colloidal AuNPs were highly efficient as catalyst for the reduction of $\mathrm{p}$ nitrophenol to $\mathrm{p}$-aminophenol at room temperature as evident from the spectrophotometric kinetic studies. As A. nilotica leaf extract has tremendous medicinal significance and AuNPs are biocompatible, the results described here will be useful in biomedical applications as well as nanoscience and nanotechnology.

\section{Additional file}

Additional file 1: Figures S1 to S5. Figure S1. Mass spectrum of Acacia nilotica leaf extract. Figure S2. High-resolution transmission electron microscopy images of AuNPs obtained with the leaf extract of Acacia nilotica at (a) 200 and (b) $60 \mathrm{mgL}^{-1}$ concentration. Figure S3. Energy disperse X-ray (EDX) spectra of AuNP derived from Acacia nilotica leaf extract $\left(60 \mathrm{mgL}^{-1}\right)$. Figure S4. X-ray diffraction pattern of AuNPs synthesized from $\mathrm{HAuCl}_{4}$ solution $(0.42 \mathrm{mM})$ using the leaf extract of Acacia nilotica. Figure S5. In A vs time plot ( $A=$ absorbance of 4-nitrophenolate), (a) with $0.2 \mathrm{~mL}$ AuNP colloid (60 $\mathrm{mgL}^{-1}$ leaf extract) and (b) with $0.4 \mathrm{~mL}$ AuNP colloid $\left(60 \mathrm{mgL}^{-1}\right.$ leaf extract). From the slopes, the apparent catalytic rate constants $(k)$ were calculated to be $0.35 \mathrm{~min}^{-1}$ in (a) and $0.42 \mathrm{~min}^{-1}$ in (b).

\section{Competing interests}

The authors declare that they have no competing interests.

\section{Authors' contributions}

RM carried out the experiments, analyzed the data and participated in the manuscript preparation. BGB as the corresponding author participated throughout the investigations and manuscript writing. NM carried out preliminary studies.

\section{Acknowledgements}

BGB thanks CSIR for funding. RM thanks UGC, New Delhi for senior research fellowship.

Received: 4 May 2013 Accepted: 12 August 2013

Published: 27 Sep 2013

\section{References}

1. Alkilany, AM, Lohse, SE, Murphy, CJ: The gold standard: gold nanoparticle libraries to understand the nano bio interface. Acc. Chem. Res. 46, 650-661 (2013)

2. Zhang, $Y$, Cui, $X$, Shi, F, Deng, $Y$ : Nano-gold catalysis in fine chemical synthesis. Chem. Rev. 112, 2467-2505 (2012)

3. Murphy, CJ, Gole, AM, Stone, JW, Sisco, PN, Alkilany, AM, Goldsmith, EC, Baxter, SC: Gold nanoparticles in biology: beyond toxicity to cellular imaging. Acc. Chem. Res. 41, 1721-1730 (2008)

4. Thomas, KG, Kamat, PV: Chromophore-functionalized gold nanoparticles. Acc. Chem. Res. 36, 888-898 (2003)

5. Anastas, PT, Kirchhoff, MM: Origins, current status, and future challenges of green chemistry. Acc. Chem. Res. 35, 686-694 (2002)

6. Nadagouda, MN, Varma, RS: Green synthesis of silver and palladium nanoparticles at room temperature using coffee and tea extract. Green Chem. 10, 859-862 (2008)

7. Dash, SS, Bag, BG: Synthesis of gold nanoparticles using renewable Punica granatum juice and study of its catalytic activity. Appl Nanosci (2013). doi:10.1007/s13204-012-0179-4

8. Gangula, A, Podila, R, Ramakrishna, M, Karanam, L, Janardhana, C, Rao, AM: Catalytic reduction of 4-nitrophenol using biogenic gold and silver nanoparticles derived from Breynia rhamnoides. Langmuir 27, 15268-15274 (2011)

9. Dash, SS, Majumdar, R, Sikder, AK, Bag, BG, Patra, BK: Saraca indica bark extract mediated green synthesis of polyshaped gold nanoparticles and its application in catalytic reduction. Appl Nanosci. (2013). doi:10.1007/s13204-013-0223-z

10. Punuri, JB, Sharma, P, Sibyala, S, Tamuli, R, Bora, U: Piper betle-mediated green synthesis of biocompatible gold nanoparticles. International Nano Letters 2, 18 (2012)

11. Bag, BG, Garai, C, Majumdar, R, Laguerre, M: Natural triterpenoids as renewable nanos. Struct. Chem. 23, 393-398 (2012)

12. Bag, BG, Dash, SS: First self-assembly study of betulinic acid, a renewable nano-sized, 6-6-6-6-5 pentacyclic monohydroxy triterpenic acid. Nanoscale 3, 4564-4566 (2011)

13. Bag, BG, Majumdar, R: Self-assembly of a renewable nano-sized triterpenoid 18ß-glycyrrhetinic acid. RSC Advances 2, 8623-8626 (2012)

14. Bag, BG, Paul, K: Vesicular and fibrillar gels by self-assembly of nanosized oleanolic acid. Asian J. Org. Chem 1, 150-154 (2012)

15. Wisdom, GOS, Shittu, GA: In vitro antimicrobial and phytochemical activities of Acacia nilotica leaf extract. J. Med. Plants Res. 4, 1232-1234 (2010)

16. Gowri, SS, Pavitha, S, Vasantha, K: Free radical scavenging capacity and antioxidant activity of young leaves and barks of Acacia nilotica (L.). Del. Int. J. Pharm. Pharm. Sci 3, 160-164 (2011)

17. Huang, $X, W u, H$, Liao, $X$, Shia, B: One-step, size-controlled synthesis of gold nanoparticles at room temperature using plant tannin. Green Chem. 12, 395-399 (2010)

18. Wunder, S, Lu Albrecht, M, Ballauff, M: Catalytic activity of faceted gold nanoparticles studied by a model reaction: evidence for substrate-induced surface restructuring. ACS Catal 1, 908-916 (2011)

$10.1186 / 2228-5326-3-53$

Cite this article as: Majumdar et al: Acacia nilotica (Babool) leaf extract mediated size-controlled rapid synthesis of gold nanoparticles and study of its catalytic activity. International Nano Letters 2013, 3:53 